\title{
Systematic review of models assessing the economic value of routine varicella and herpes zoster vaccination in high-income countries
}

\author{
Oliver Damm*, Bernhard Ultsch², Johannes Horn ${ }^{3}$, Rafael T. Mikolajczyk ${ }^{3,4}$, Wolfgang Greiner ${ }^{1}$ and Ole Wichmann²
}

\begin{abstract}
Background: A systematic review was conducted to assess the cost-effectiveness of routine varicella and herpes zoster ( $\mathrm{HZ}$ ) vaccination in high-income countries estimated by modelling studies.

Methods: A PubMed search was performed to identify relevant studies published before October 2013. Studies were included in the review if they (i) evaluated the cost-effectiveness of routine childhood or adolescent varicella vaccination and/or $\mathrm{HZ}$ vaccination targeting the elderly, and if they (ii) reported results for high-income countries.

Results: A total of 38 model-based studies were identified that fulfilled the inclusion criteria. Routine childhood or adolescent varicella vaccination was cost-effective or cost-saving from a payer perspective and always cost-saving from a societal perspective when ignoring its potential impact on $\mathrm{HZ}$ incidence due to reduced or absent exogenous boosting. The inclusion of the potential impact of childhood varicella vaccination on $\mathrm{HZ}$ led to net quality-adjusted life-year (QALY) losses or incremental cost-effectiveness ratios exceeding commonly accepted thresholds. Additional $\mathrm{HZ}$ vaccination could partially mitigate this effect. Studies focusing only on the evaluation of $\mathrm{HZ}$ vaccination reported a wide range of results depending on the selected target age-group and the vaccine price, but most found $\mathrm{HZ}$ vaccination to be a cost-effective or marginally cost-effective intervention. Cost-effectiveness of $\mathrm{HZ}$ vaccination was strongly dependent on the age at vaccination, the price of the vaccine, the assumed duration of protection and the applied cost per QALY threshold.
\end{abstract}

Conclusions: While $\mathrm{HZ}$ vaccination is mostly considered cost-effective, cost-effectiveness of varicella vaccination primarily depends on the in- or exclusion of exogenous boosting in the model. As a consequence, clarification on the role of exogenous boosting is crucial for decision-making regarding varicella vaccination.

Keywords: Cost-effectiveness, Economic evaluation, Vaccination, Varicella, Zoster, Systematic review

\section{Background}

Primary infection with varicella-zoster virus (VZV) causes varicella (chickenpox), which occurs mainly in childhood $[1,2]$. The virus persists lifelong in the dorsal roots of the spinal and cranial ganglia. Later in life the virus can be reactivated, manifesting as shingles (herpes zoster, HZ), a painful skin rash that lasts approximately one month $[1,3]$. The main complication of $\mathrm{HZ}$ is postherpetic neuralgia $(\mathrm{PHN})$, a long lasting neuropathic pain in the area formerly affected by the $\mathrm{HZ}$ rash [3-6].

\footnotetext{
* Correspondence: oliver.damm@uni-bielefeld.de

'Department of Health Economics and Health Care Management, Bielefeld

School of Public Health, Bielefeld University, Bielefeld, Germany

Full list of author information is available at the end of the article
}

Live-attenuated monovalent varicella vaccines or combination vaccines against measles, mumps, rubella and varicella (MMRV) licensed for use in children are available in most industrialised countries. As of today there is one $\mathrm{HZ}$ vaccine licensed for individuals aged 50 years and older.

Routine childhood varicella vaccination is generally recommended in the United States, Australia, Canada, Qatar, Saudi Arabia, Republic of Korea, Taiwan, Uruguay and several countries in Europe including Germany, Greece, Finland and parts of Italy and Spain [7, 8]. A significant decline in varicella incidence was observed after the introduction of routine vaccination in several countries [8-11]. Nationwide vaccination recommendations

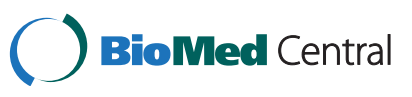


for the prevention of $\mathrm{HZ}$ currently exist in Austria for individuals aged 50+ [12], the United States and in Canada for individuals aged $60+[13,14]$ and in the UK for individuals aged 70-79 [15].

In the 1960s, Hope-Simpson hypothesised that after primary infection a re-exposure to wild-type VZV would sub-clinically boost the individual's VZV-specific immunity, thereby suppressing VZV reactivation and decreasing the probability of developing HZ [16]. Since then, the hypothesis that $\mathrm{HZ}$ incidence will increase in a population with routine varicella vaccination has been discussed in the literature [17]. According to models, varicella vaccination might lead to a substantial increase in HZ incidence during approximately $40-50$ years after initiation of routine vaccination $[18,19]$. In the United States, where routine varicella vaccination was introduced in 1995, studies monitoring HZ incidence have reported inconsistent results until today [20]. However, a more recent systematic review based on 39 multidisciplinary studies concluded that exogenous boosting exists, but its extent and public health impact remain unclear [21].

Our objective was to assess the cost-effectiveness of routine varicella and $\mathrm{HZ}$ vaccination in high-income countries estimated by modelling studies.

\section{Methods}

\section{Search strategy}

A PubMed search was performed to identify Englishand German-language articles on economic evaluations of varicella and $\mathrm{HZ}$ vaccination published before October 2013. The systematic literature search was conducted using the following key words: (varicella OR chickenpox OR herpes zoster OR shingles OR varicella-zoster OR VZV OR "Chickenpox" [MeSH] OR "Herpes Zoster" $[\mathrm{MeSH}]$ ) AND (vaccination OR vaccine OR vaccinating OR vaccinate $O R$ vaccinated $O R$ immunisation $O R$ immunization OR "Vaccination" [MeSH] OR "Vaccine" [MeSH] OR "Chickenpox Vaccine" [MeSH] OR "Herpes Zoster Vaccine" $[\mathrm{MeSH}]$ ) AND (economic OR economics OR cost OR costs OR cost-effectiveness OR cost-effective OR cost-utility OR cost-benefit OR benefit-cost OR costsaving OR pharmacoeconomic OR pharmacoeconomics OR ICER OR QALY OR "Costs and Cost Analysis" [MeSH]). Application of the non-MeSH search terms was restricted to titles and abstracts of the PubMed records. In addition, we screened reference lists of all included studies to identify further articles of interest.

\section{Study selection}

Titles and abstracts of the obtained search results were screened independently by two reviewers. Full-text versions of all potentially relevant studies were retrieved and assessed according to pre-defined inclusion and exclusion criteria by the same two reviewers. Any disagreements between reviewers on inclusion of particular studies were resolved by consensus. A study was included if it was a full or partial economic evaluation of routine childhood (or adolescent) varicella vaccination or a $\mathrm{HZ}$ immunisation scheme targeting the elderly and if the modelling study reported results for a high-income country as specified by the World Bank (high-income OECD-members) [22]. Inclusion criteria related to comparators included no vaccination as well as existing vaccination programmes or private coverage. We did not define inclusion or exclusion criteria related to outcome measures. We excluded nonoriginal research papers (i.e. review articles, letters, and editorials), studies that focused on vaccination of specific target groups (e.g. health care workers, adults without history of chickenpox, transplant patients, seronegative postpartum women, army recruits and cadets, immigrants and refugees), studies that did not provide sufficient details on the applied methods, studies that evaluated only combined strategies of serotesting and vaccination as well as studies that evaluated combination vaccines without reporting separate results for each component.

\section{Data extraction and synthesis}

Critical appraisal of all included studies was undertaken by using the framework for quality assessment of decision-analytic models proposed by Philips et al. [23]. The quality assessment was performed by two independent reviewers. The framework used considers aspects related to structure, data, and consistency of health economic models. The following information was systematically extracted from each included study: citation details, country, characteristics of the vaccination programme (e.g. target age group, vaccine type, vaccine efficacy, vaccination coverage), main features of the modelling approach (e.g. model type, time horizon, interaction between varicella and HZ), characteristics of the economic analysis (e.g. determination of the perspective, choice of discount rate, valuation of health gains), key findings as well as funding sources. The economic value of routine varicella and $\mathrm{HZ}$ vaccination was assessed by comparing incremental costeffectiveness ratios (ICERs) and/or benefit-cost ratios (BCRs) among studies taking into account different immunisation strategies, perspectives as well as clinical and epidemiological features of VZV.

To improve comparability between studies and across countries, all cost estimates were inflated to 2010 values (latest price year used in included studies) applying country-specific consumer price indices and converted to Euros with the German level of purchasing power using purchasing power parities obtained from the Organisation for Economic Co-operation and Development (OECD) [24].

The reporting of this systematic review was performed in accordance with the PRISMA statement [25]. However, 
not all items of the PRISMA statement checklist are applicable to economic evaluations.

\section{Results}

\section{Search results and quality assessment}

The literature search in PubMed identified 351 articles. After screening the titles and abstracts of these hits, 92 papers were considered for full-text review. 41 papers of the obtained full-text articles met the inclusion criteria. The main reasons for exclusion were incorrect type of study (such as review articles), incorrect intervention or studies assessing the vaccination of specific target groups. Few studies were excluded due to insufficient information on the methods used. Four of the 41 papers that met the inclusion criteria reported on the same modelling study and three of them were therefore excluded. Finally, 38 studies were included in the review. A flow chart of the study selection process and the corresponding results is outlined in Fig. 1.

Most of the included studies were of high quality. However, some studies evaluating varicella vaccination did not use a dynamic modelling approach and hence are not able to capture indirect effects of vaccination. Most studies performed probabilistic sensitivity analysis to address the issue of parameter uncertainty, but only a few studies evaluated the impact of structural uncertainties. Several studies also lack detailed descriptions/discussion of the sources of utility weights.

\section{Study characteristics Varicella vaccination}

We included 23 model-based studies evaluating the cost-effectiveness of routine varicella vaccination. Table 1 gives an overview of general study characteristics and information on the applied modelling framework of these studies. The majority of the studies was performed for European countries.

13 studies used a fully dynamic modelling approach in terms of simulating the transmission dynamics of varicella. 10 studies were based on static models. By definition, all dynamic models accounted for herd protection effects. The static model from Israel reported to have included partial herd protection effects by use of an adjustment factor. Apart from herd protection effects, varicella immunisation programmes can induce other populationlevel effects such as the hypothesised increase in HZ incidence due to a reduced or absent exogenous boosting after varicella vaccine introduction and subsequent decrease in wild virus circulation in the population. Most of the models ignored the close relationship between varicella and HZ. Only four studies modelled the possible impact of routine varicella vaccination on $\mathrm{HZ}$ incidence in a population due to a decrease in wild-type VZV circulation.

Many studies performed a cost comparison analysis and reported the results as BCRs. Some of these studies claimed to have conducted a cost-benefit analysis but this would require a monetary valuation of health effects.

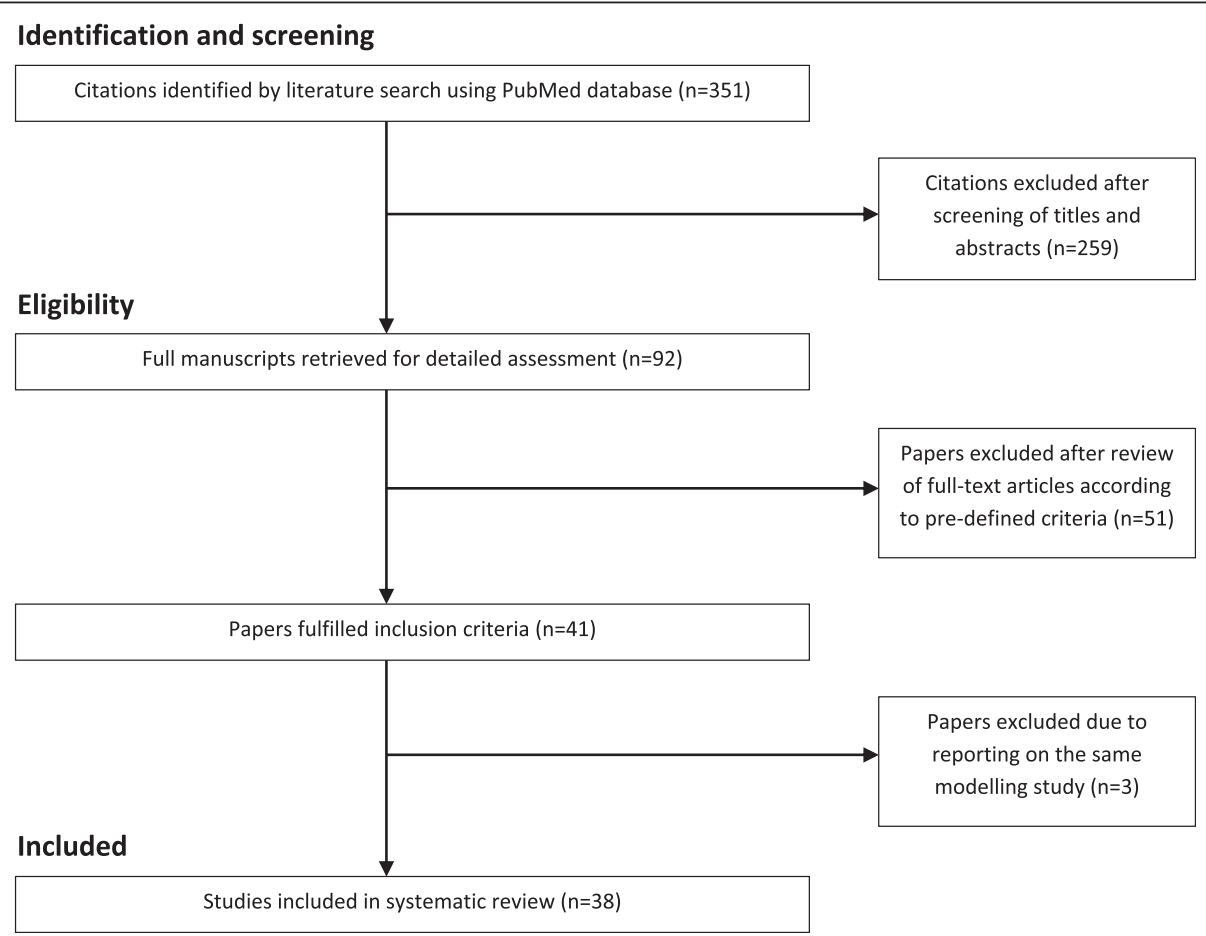

Fig. 1 Flow chart of study selection process 
Table 1 General study characteristics of the models evaluating routine varicella vaccination

\begin{tabular}{|c|c|c|c|c|c|c|c|c|c|c|}
\hline Study & Country & $\begin{array}{l}\text { Model } \\
\text { type }\end{array}$ & $\begin{array}{l}\text { Accounting } \\
\text { for herd } \\
\text { protection }\end{array}$ & $\begin{array}{l}\text { Impact of varicella } \\
\text { vaccination on } \\
\text { HZ incidence }\end{array}$ & $\begin{array}{l}\text { Time } \\
\text { horizon }\end{array}$ & $\begin{array}{l}\text { Type of } \\
\text { economic } \\
\text { evaluation }\end{array}$ & Perspective & $\begin{array}{l}\text { Discount rate } \\
\text { (costs/health } \\
\text { effects) }\end{array}$ & $\begin{array}{l}\text { Costing year } \\
\text { and currency }\end{array}$ & $\begin{array}{l}\text { Funding } \\
\text { source }\end{array}$ \\
\hline Banz et al. [35] & Germany & Dynamic & Yes & No & 30 years & CC & Health care payer; societal & $5 \% / 0 \%$ & 1999 EUR & Industry \\
\hline Banz et al. [51] & Switzerland & Dynamic & Yes & No & 30 years & CC; CEA & Health care payer; societal & $5 \% / 0 \%$ & 2008 CHF & Industry \\
\hline Beutels et al. [37] & Germany & Static & No & No & 70 years & CC; CEA & Health care payer; societal & $5 \% / 5 \%$ & 1995 DEM & Independent \\
\hline Bilcke et al. [30] & Belgium & Dynamic & Yes & $\begin{array}{l}\text { Depending on the } \\
\text { type of analysis }\end{array}$ & Various & CEA; CUA & Health care payer & $3 \% / 1.5 \%$ & $2010^{\mathrm{a}}$ EUR & Independent \\
\hline Bonanni et al. [36] & Italy & Dynamic & Yes & No & 30 years & CC & Health care payer; societal & $3 \% / 0 \%$ & $2007^{a}$ EUR & Industry \\
\hline Brisson et al. [31] & Canada & Dynamic & Yes & $\begin{array}{l}\text { Yes (only in a } \\
\text { separate analysis) }\end{array}$ & 30 years & $C C ; C E A$ & Health care payer; societal & $3 \% / 3 \%$ & 1997/1998 CAD & Independent \\
\hline Brisson et al. [32] & England and Wales & Dynamic & Yes & Yes & 80 years & CUA & Health care payer; societal & $3 \% / 3 \%$ & 2001 GBP & Independent \\
\hline Coudeville et al. [52] & France & Dynamic & Yes & No & 30 years & CC & Health care payer; societal ${ }^{b}$ & $5 \% / N A$ & 1995 FRF & Industry \\
\hline Coudeville et al. [53] & Italy & Dynamic & Yes & No & 50 years & $\mathrm{CC}$ & Health care payer; societal & $3 \% / N A$ & 2002 EUR & Industry \\
\hline Coudeville et al. [54] & France and Germany & Dynamic & Yes & No & 50 years & CC; CEA & Health care payer; societal & $3 \% / 3 \%$ & 2002 EUR & Industry \\
\hline Diez Domingo et al. [55] & Spain & Static & No & No & 20 years & CC & Health care payer; societal & $5 \% / N A$ & 1994 PTA & Independent \\
\hline Getsios et al. [56] & Canada & Static & No & No & 70 years & CEA; CUA & Health care payer; societal & $3 \% / 3 \%$ & 1998 CAD & Independent \\
\hline Ginsberg \& Somekh [57] & Israel & Static & Partially & No & Lifetime & CC & Health care payer; societal & $3 \% / N A$ & 2002 USD & Independent \\
\hline Hammerschmidt et al. [58] & Germany & Dynamic & Yes & No & 30 years & CC & Health care payer; societal & $\begin{array}{l}\text { Not specified } \\
\text { (probably } 5 \% \\
\text { for costs) }\end{array}$ & 1999/2006 EUR & Industry \\
\hline Huse et al. [59] & USA & Static & No & No & 24 years & $\mathrm{CC}$ & Societal & $5 \% / 5 \%$ & 1991 USD & Industry \\
\hline Lenne et al. [60] & Spain & Dynamic & Yes & No & 50 years & CC; CEA & Health care payer; societal & $3 \% / 0 \%$ & 2004 EUR & Industry \\
\hline Lieu et al. [61] & USA & Dynamic & Yes & No & 30 years & CC; CEA & Health care payer; societal & $5 \% / 5 \%$ & 1990 USD & Independent \\
\hline Preblud et al. [62] & USA & Static & No & No & 30 years & $C C$ & Health care payer; societal & $5 \% / 0 \%$ & 1984 USD & Independent \\
\hline Scuffham et al. [63] & New Zealand & Static & No & No & 30 years & $\mathrm{CC}$ & Health care payer; societal & $5 \% / 5 \%$ & 1997 NZD & Industry \\
\hline Scuffham et al. [34] & Australia & Static & No & No & 30 years & CEA & Health care payer & $5 \% / 5 \%$ & 1996/1997 AUD & Independent \\
\hline Thiry et al. [64] & Italy & Static & No & No & 100 years & CC; CEA & Health care payer; societal & $3 \% / 3 \%$ & 2002 EUR & Industry \\
\hline van Hoek et al. [33] & UK & Dynamic & Yes & Yes & Infinite & CUA & Health care payer & $3.5 \% / 3.5 \%$ & 2007 GBP & Independent \\
\hline Zhou et al. [29] & USA & Static & No & No & Lifetime & CC; CUA & Health care payer; societal & $3 \% / 3 \%$ & 2006 USD & Industry \\
\hline
\end{tabular}

\section{CC cost-comparison; CEA cost-effectiveness analysis; CUA cost-utility analysis; $N A=$ not applicable}

${ }^{\text {a Assumption }}$

${ }^{b}$ Indirect costs were not evaluated in monetary terms but reported as the number of days of absence from work

'Personal communication with the author (1999 prices; 2006 vaccine prices) 
However, the included studies which we classified as cost comparison analyses only considered costs and cost offsets instead of valuing health effects in monetary terms.

Most of the studies adopted both a health care payer and a societal perspective. Costs were discounted with a discount rate ranging from 3 to $5 \%$. The discount rate for health effects ranged from 0 to $5 \%$. Vaccination costs varied widely across studies. The simulated time horizons ranged from 20 years to a lifetime or an infinite time horizon, but most studies used a period of 30 or 50 years.

Aspects related to vaccine characteristics and immunisation strategies considered in the included studies are presented in Table 2 . The majority of the studies compared universal vaccination to a situation without vaccination. Few studies chose an existing one-dose varicella vaccination programme or a situation with low private vaccination coverage as comparator. While most of the studies considered a 1-dose vaccination schedule, some studies also (or exclusively) assessed the impact of two varicella vaccine doses. Considered vaccine efficacy ranged from 80 to $97 \%$ and from 93 to $96 \%$ for the first and the second dose, respectively. Waning of vaccine-induced immunity was modelled in very different ways. Some models did not consider waning immunity at all; other models used yearly waning rates of 0.5 or $3.1 \%$. A UK study used various waning rates ranging from 0.05 to $6.7 \%$. Some studies stated that waning was applied to $15 \%$ of the protected vaccinees without quantifying the waning rate per year. Assumed vaccination coverage in the models ranged from 30 to $97.15 \%$. Most of the studies included a fee for administering the vaccine. Some studies included additional costs for treating adverse events and/or costs of vaccine wastage.

\section{Herpes zoster vaccination}

We included 17 model-based studies evaluating the costeffectiveness of $\mathrm{HZ}$ vaccination. The main study characteristics are summarised in Table 3. We identified 15 studies which considered the health economic impact of $\mathrm{HZ}$ vaccination exclusively. The remaining two studies considered both varicella and $\mathrm{HZ}$ vaccination. Most studies were conducted for European countries. The two studies targeting varicella as well as $\mathrm{HZ}$ vaccination applied a dynamic modelling approach. One study used a discrete-event simulation model that simulated individual patients [26]. All other cost-effectiveness studies were based on static (Markov) state-transition models or similar models using single or multiple cohorts. However, the number of included disease states differed among models. While most models considered common states like healthy, HZ, PHN, and death, four studies included also different pain levels (mild, moderate and severe) for HZ and PHN. Some studies failed to report explicit information on the modelled health states or their number.
All studies conducted a cost-utility analysis (CUA). Furthermore, almost half of the studies also performed a cost-effectiveness analysis (CEA). According to respective national guidelines, six studies used different discount rates for costs and health effects. One study used equal discount rates for costs and health effects that changed over time following the current French guidelines: A $4 \%$ discount rate was used for the first 30 years of the model run and afterwards the discount rate was reduced to $2 \%$. Most studies used a lifetime horizon.

Details of the $\mathrm{HZ}$ vaccination-related input data are shown in Table 4. All models compared a vaccination scenario with no vaccination. Vaccine efficacy estimates were mostly based on clinical trial data of the Shingles Prevention Study [27]. Several studies neglected waning of vaccine-induced immunity in the base-case analysis. The age at vaccination varied between 50 and 80 years. Six studies did not report the assumed vaccination coverage. However, in static models vaccination costs and effects are proportional to vaccination coverage and hence the level of coverage has no impact on the ICER.

A wide range of vaccination costs was applied across the studies. One study [28] considered vaccination costs between EUR 43.85 and 438.46 per dose in multiple scenarios, while the vaccination costs among the other studies ranged from EUR 81.54 to 147.48 per dose or per immunisation course. Thirteen studies included administration fees in these cost estimates. One study included additional costs for a public awareness campaign, patient time costs, and costs for treating adverse events.

\section{Results of the included studies Varicella vaccination}

The results of economic evaluations of varicella vaccination are summarised in Table 5. BCRs for one-dose varicella vaccination of young children ranged between 0.30 and 1.94 when taking a health care payer perspective and ignoring a potential impact on HZ. Six studies reported BCRs above 1 or stated that vaccination would lead to cost-savings. Eleven studies reported BCRs below 1 or calculated ICERs. In these studies, costs per life year gained (LYG) ranged between EUR 563 and EUR 40,193. One study reported a BCR of 1 which means that onedose varicella vaccination was a cost-neutral intervention [29]. When adopting a societal perspective and ignoring a potential impact on $\mathrm{HZ}$, all studies found that one-dose varicella vaccination of toddlers and young children would be cost-saving with BCRs ranging from 1.61 to 19.33.

BCRs for two dose-vaccination targeting young children ranged between 0.13 and 1.08 when adopting a health care payer perspective and between 0.56 and 3.47 when taking a societal perspective. The study by Bilcke et al. [30] reported ICERs below EUR 35,000 per QALY for a two dose-vaccination scheme from a payer perspective when 
Table 2 Vaccine characteristics and immunisation strategies considered in the models evaluating routine varicella vaccination

\begin{tabular}{|c|c|c|c|c|c|}
\hline Study & Age at vaccination & Vaccine efficacy & Waning (per year) & Vaccination coverage & $\begin{array}{l}\text { Vaccination costs per dose } \\
\text { (2010 EUR; German price level) }\end{array}$ \\
\hline Banz et al. [35] & 15 months; $11-12$ years & $86 \%$ & $0.5 \%$ & $\begin{array}{l}85 \% \text { (children); } 30 \% \\
\text { (adolescents) }\end{array}$ & $\begin{array}{l}\text { EUR } 65.93 \text { (children) }^{\mathrm{a}} \text {; } \\
\text { EUR } 71.38 \text { (adolescents) }\end{array}$ \\
\hline Banz et al. [51] & $1-2$ years; $11-15$ years & $95 \%$ & $0.5 \%$ & $\begin{array}{l}70 \% \text { (children); } 85 \% \\
\text { (adolescents) }\end{array}$ & $\begin{array}{l}\text { EUR } 42.20 \text { (children) }^{\mathrm{a}} \text {; } \\
\text { EUR } 56.65 \text { (adolescents) }^{\text {a }}\end{array}$ \\
\hline Beutels et al. [37] & 15 months; 12 years & $90 \%$ & $\begin{array}{l}\text { Waning in } 15 \% \text { of the } \\
\text { protected vaccinees }\end{array}$ & $70 \%$ & EUR $50.82^{\mathrm{a}}$ \\
\hline Bilcke et al. [30] & $\begin{array}{l}1 \text { year (1st dose); } 4,6 \text { or } \\
11 \text { years ( } 2 \text { nd dose) }\end{array}$ & $\begin{array}{l}\text { Data from van Hoek } \\
\text { et al. } 2012\end{array}$ & $\begin{array}{l}\text { Data from van Hoek } \\
\text { et al. } 2012\end{array}$ & $\begin{array}{l}50 \% \text { or } 95 \% \text { ( } 1 \text { st dose); } \\
50 \%, 80 \% \text { or } 90 \% \\
\text { (2nd dose) }\end{array}$ & EUR $44.92^{\mathrm{a}}$ \\
\hline Bonanni et al. [36] & 12-18 months; 13 years & $\begin{array}{l}90 \% \text { (1st dose); } \\
93 \% \text { (2nd dose) }\end{array}$ & $3 \%$ & $85 \%$ & EUR $46.81^{\mathrm{a}, \mathrm{c}}$ \\
\hline Brisson et al. [31] & 12 months; 12 years & $93 \%$ & $3.1 \%$ & $\begin{array}{l}90 \% \text { (infants); } 80 \% \\
\text { (adolescents) }\end{array}$ & $\begin{array}{l}\text { EUR } 51.42 \text { (children); } \\
\text { EUR } 68.57 \text { (adolescents) }\end{array}$ \\
\hline Brisson et al. [32] & Infants; 11 years & $93 \%$ & $3.1 \%$ & $\begin{array}{l}90 \% \text { (infants); } 80 \% \\
\text { (adolescents) }\end{array}$ & $\begin{array}{l}\text { EUR } 44.32 \text { (children) })^{a} \text {; } \\
\text { EUR } 59.10 \text { (adolescents) }\end{array}$ \\
\hline Coudeville et al. [52] & $<6$ years & $90 \%$ & $\begin{array}{l}\text { Waning in } 15 \% \text { of the } \\
\text { protected vaccinees }^{b}\end{array}$ & $80 \%$ & EUR $18.11^{c, d}$ \\
\hline Coudeville et al. [53] & $12-36$ months & $97 \%$ & $3.1 \%$ & $45 \%-90 \%$ & EUR $52.00^{\mathrm{a}, \mathrm{c}}$ \\
\hline Coudeville et al. [54] & $12-36$ months & $97 \%$ & $3.1 \%$ & $45 \%-90 \%$ & $\begin{array}{l}\text { EUR } 64.08 \text { (Germany) })^{a, c} ; \\
\text { EUR } 59.56 \text { (France) })^{\mathrm{a}, \mathrm{c}}\end{array}$ \\
\hline Diez Domingo et al. [55] & 15 months & $90 \%$ & No waning & $95 \%$ & EUR 37.11 \\
\hline Getsios et al. [56] & 12 months & $90 \%$ & $\begin{array}{l}\text { Waning in } 15 \% \text { of the } \\
\text { protected vaccinees }{ }^{b}\end{array}$ & $85 \%$ & EUR $60.25^{a, d}$ \\
\hline Ginsberg \& Somekh [57] & 12 months & $87.6 \%$ & $3.1 \%$ & $94 \%$ & EUR $8.01^{\mathrm{a}, \mathrm{c}, \mathrm{d}}$ \\
\hline Hammerschmidt et al. [58] & $\begin{array}{l}\text { 11-23 months } \\
\text { (catch-up of } 2-17 \text { year olds) }\end{array}$ & $\begin{array}{l}86 \% \text { (1-dose schedule); } \\
95 \% \text { (2-dose schedule) }\end{array}$ & $0.5 \%$ & $\begin{array}{l}90 \% \text { (1st dose); } 80 \% \\
\text { (2nd dose); } 30 \% \text { (catch-up); } \\
10 \% \text { (comparator) }\end{array}$ & $\begin{array}{l}\text { EUR } 47.38 \text { (monovalent vaccine); } \\
\text { EUR } 47.92 \text { (varicella-attributable } \\
\text { cost of the MMRV vaccine) }\end{array}$ \\
\hline Huse et al. [59] & 15 months & $95 \%$ & No waning & Not specified & EUR $62.31^{\mathrm{a}}$ \\
\hline Lenne et al. [60] & $1-2$ years & $97 \%$ & $3,1 \%$ & $97,15 \%$ & EUR $42.54^{\mathrm{a}}$ \\
\hline Lieu et al. [61] & $<6$ years & $90 \%$ & $\begin{array}{l}\text { Waning in } 15 \% \text { of the } \\
\text { protected vaccinees }\end{array}$ & $97 \%$ & EUR $54.12^{\mathrm{a}}$ \\
\hline Preblud et al. [62] & 15 months & $90 \%$ & No waning & $90 \%$ & EUR 25.53 \\
\hline Scuffham et al. [63] & 15 months & $95 \%$ & No waning & $80 \% ; 10 \%$ (comparator) & EUR 45.94 \\
\hline
\end{tabular}


Table 2 Vaccine characteristics and immunisation strategies considered in the models evaluating routine varicella vaccination (Continued)

\begin{tabular}{|c|c|c|c|c|c|}
\hline Scuffham et al. [34] & 12 months; 12 years & $95 \%$ & No waning & $\begin{array}{l}80 \% \text { (infants); } 50-75 \% \\
\text { (adolescents) }\end{array}$ & EUR 42.26 \\
\hline Thiry et al. [64] & 11 years & $93,12 \%$ & $3.1 \%$ & $60 \%$ & EUR $52.36^{a}$ \\
\hline van Hoek et al. [33] & $\begin{array}{l}1 \text { year ( } 1 \text { st dose); } \\
3 \text { years ( } 2 \text { nd dose) }\end{array}$ & $\begin{array}{l}89-96 \% \text { (1st dose); } \\
93-96 \% \text { (2nd dose) }\end{array}$ & $\begin{array}{l}1.5-6.7 \% \text { (1st dose); } \\
0.05-2.6 \% \text { (2nd dose) }\end{array}$ & $\begin{array}{l}90 \% \text { (1st dose); } \\
80 \% \text { (2nd dose) }\end{array}$ & EUR 41.19 \\
\hline Zhou et al. [29] & Children & $\begin{array}{l}80 \% \text { (1-dose schedule) } \\
93 \% \text { (2-dose schedule) }\end{array}$ & No waning & $\begin{array}{l}\text { Age-specific coverage rates; } \\
95 \% \text { ( } 2 \text { nd dose) }\end{array}$ & $\begin{array}{l}\text { EUR } 49.90 \text { (monovalent vaccine) }^{f} \text {; } \\
\text { EUR } 65.64 \text { (MMRV vaccine) }^{f}\end{array}$ \\
\hline
\end{tabular}

MMRV measles, mumps, rubella and varicella

ancluding administration costs

bWaning rate per year not quantified

Including costs of treating adverse events

Including vaccine wastage

eThis efficacy estimate was not directly used in the model; the vaccine-induced reduction in incidence was calculated by using age-specific surveillance data fPublic sector price 
Table 3 General study characteristics of the models evaluating routine $\mathrm{HZ}$ vaccination

\begin{tabular}{|c|c|c|c|c|c|c|c|c|}
\hline Study & Country & $\begin{array}{l}\text { Model } \\
\text { type }\end{array}$ & Time horizon & $\begin{array}{l}\text { Type of } \\
\text { economic } \\
\text { evaluation }\end{array}$ & Perspective & $\begin{array}{l}\text { Discount rate } \\
\text { (costs/health effects) }\end{array}$ & $\begin{array}{l}\text { Costing year } \\
\text { and currency }\end{array}$ & Funding source \\
\hline Annemans et al. [65] & Belgium & Static & Lifetime & CEA; CUA & $\begin{array}{l}\text { Health care payer } \\
\text { (with and without co-payments); societal }\end{array}$ & $3 \% / 1.5 \%$ & 2007 EUR & Industry \\
\hline Bilcke et al. [38] & Belgium & Static & Lifetime & CEA; CUA & Health care payer & $3 \% / 1.5 \%$ & $2009^{\mathrm{a}}$ EUR & Independent \\
\hline Bilcke et al. [30] & Belgium & Dynamic $^{b}$ & Various & CEA; CUA & Health care payer & $3 \% / 1.5 \%$ & $2011^{a}$ EUR & Independent \\
\hline Bresse et al. [66] & France & Static & Lifetime & CEA; CUA & $\begin{array}{l}\text { Health care payer } \\
\text { (with and without co-payments) }\end{array}$ & $4 \% / 4 \%^{c}$ & 2010 EUR & Industry \\
\hline Brisson et al. [67] & Canada & Static & Lifetime & CUA & Health care payer & $5 \% / 5 \%$ & 2005 CAD & Industry \\
\hline de Boer et al. [40] & Netherlands & Static & Up to 41 years & CUA & Health care payer; societal & $4 \% / 1.5 \%$ & 2010 EUR & Independent \\
\hline Edmunds et al. [68] & England and Wales & Static & Lifetime & CEA; CUA & Health care payer & $3 \% / 3 \%$ & 1998 GBP & Independent \\
\hline Hornberger et al. [28] & USA & Static & 30 years & CUA & Societal & $3 \% / 3 \%$ & 2006 USD & Independent \\
\hline Moore et al. [69] & UK & Static & Lifetime & CEA; CUA & Health care payer; societal & $3.5 \% / 3.5 \%$ & 2006 GBP & Industry \\
\hline Najafzadeh et al. [26] & Canada & Static & Lifetime & CUA & Health care payer & $5 \% / 5 \%$ & 2008 CAD & Independent \\
\hline Pellissier et al. [70] & USA & Static & Lifetime & CUA & Health care payer; societal & $3 \% / 3 \%$ & 2006 USD & Industry \\
\hline Rothberg et al. [42] & USA & Static & Not specified & CUA & Societal & $3 \% / 3 \%$ & 2005 USD & Independent \\
\hline Szucs et al. [71] & Switzerland & Static & Lifetime & CEA; CUA & Health care payer; societal & $3.5 \% / 1.5 \%$ & $2010^{\mathrm{a}} \mathrm{CHF}$ & Industry \\
\hline Ultsch et al. [39] & Germany & Static & Lifetime & CEA; CUA & Health care payer; societal & $3 \% / 3 \%$ & 2010 EUR & Independent \\
\hline van Hoek et al. [72] & England and Wales & Static & Lifetime & CUA & Health care payer & $3.5 \% / 3.5 \%$ & 2006 GBP & Independent \\
\hline van Hoek et al. [33] & UK & Dynamic $^{b}$ & Infinite & CUA & Health care payer & $3.5 \% / 3.5 \%$ & 2007 GBP & Independent \\
\hline van Lier et al. [41] & Netherlands & Static & Not specified & CUA & Health care payer; societal & $4 \% / 1.5 \%$ & 2008 EUR & Independent \\
\hline
\end{tabular}

CEA cost-effectiveness analysis; CUA cost-utility analysis

${ }^{2}$ Personal communication with the author or assumptio

${ }^{\mathrm{b}}$ Combined evaluation of varicella and $\mathrm{HZ}$ vaccination

'Discount rate for costs and health effects was reduced to $2 \%$ after 30 years 
Table 4 Vaccine characteristics and immunisation strategies considered in the models evaluating routine $\mathrm{HZ}$ vaccination

\begin{tabular}{|c|c|c|c|c|c|}
\hline Study & $\begin{array}{l}\text { Age at vaccination } \\
\text { (in years) }\end{array}$ & Vaccine efficacy against $\mathrm{HZ}$ & Waning or duration of protection & $\begin{array}{l}\text { Vaccination } \\
\text { coverage }^{\mathrm{a}}\end{array}$ & $\begin{array}{l}\text { Vaccination costs } \\
\text { per dose (2010 EUR, } \\
\text { German price level) }\end{array}$ \\
\hline Annemans et al. [65] & $50+$ & 37.6-63.9\%, age-dependent & No waning ${ }^{b}$ & $20 \%$ & EUR $141.39^{C}$ \\
\hline Bilcke et al. [38] & $60-85$ & Age-dependent (values are reported graphically only) & $\begin{array}{l}\text { Consideration of waning depends on the choice } \\
\text { of scenario }\end{array}$ & $30 \%$ & EUR $106.95^{\mathrm{d}}$ \\
\hline Bilcke et al. [30] & 50 or 60 & $77 \%$ & $\begin{array}{l}\text { Duration of protection of } 7.5 \text { years or } \\
\text { lifelong protection }\end{array}$ & $30 \%$ or $70 \%$ & EUR $103.38^{d}$ \\
\hline Bresse et al. [66] & $65+$ & $18-64 \%$, age-dependent & $\begin{array}{l}4.15 \% \text { per year and vaccine efficacy was set to zero } \\
\text { after } 10 \text { years }\end{array}$ & $20 \%$ & EUR $117^{f}$ \\
\hline Brisson et al. [67] & $50-80$ & $26-75 \%$, age-dependent & No waning ${ }^{b}$ & Not specified & EUR 108.60 \\
\hline de Boer et al. [40] & $60-75$ & 41.2-69.4\%, age-dependent & $8.3 \%$ per year (= duration of protection of 12 years) & Not specified & EUR $89.10^{d}$ \\
\hline Edmunds et al. [68] & 65 & $30-70 \%$ & Duration of protection of 2.5 years to life long & $60 \%$ & EUR $122.13^{\mathrm{d}, \mathrm{g}}$ \\
\hline Hornberger et al. [28] & 69 & $\begin{array}{l}\text { Modelled by applying age-specific incidence of } \mathrm{HZ} \\
\text { in vaccine and placebo-treated arm of the clinical } \\
\text { study, age-dependent }\end{array}$ & Duration of protection of 30 years & Not specified & EUR $43.85-438.46^{\mathrm{d}, \mathrm{h}}$ \\
\hline Moore et al. [69] & $50+$ & 37.6-63.9 \%, age-dependent & No waning ${ }^{b}$ & $40 \%$ & EUR $143.28^{d}$ \\
\hline Najafzadeh et al. [26] & $60+$ & $\begin{array}{l}\text { Modelled by applying age-specific incidence of } \mathrm{HZ} \\
\text { in vaccine and placebo-treated arm of the clinical } \\
\text { study, age-dependent }\end{array}$ & $4.5 \%$ per year & Not specified & EUR 101.83 \\
\hline Pellissier et al. [70] & $60+$ & 27.1-69.8\%, age-dependent & No waning ${ }^{b}$ & Not specified & EUR $147.32^{d}$ \\
\hline Rothberg et al. [42] & $60-80$ & Age-dependent & Waning considered but not quantified & Not specified & EUR $134.74^{d}$ \\
\hline Szucs et al. [71] & 70-79 & 37.60-63.9 \%, age-dependent & No waning ${ }^{\mathrm{b}}$ & $20 \%$ & EUR $143.09^{d}$ \\
\hline Ultsch et al. [39] & $50-80$ & 13.22-69.8 \%, age-dependent & $\begin{array}{l}8.3 \% \text { per year following } 10 \text { years of stable } \\
\text { vaccine efficacy }\end{array}$ & $20 \%$ & EUR $147.48^{d}$ \\
\hline van Hoek et al. [72] & $60-75$ & $\begin{array}{l}31-95 \% \text { (based on } 15 \text { different take and waning scenarios), } \\
\text { age-dependent }\end{array}$ & $\begin{array}{l}\text { Duration of protection of 3.6-100 years } \\
\text { (based on } 15 \text { different take and waning scenarios) }\end{array}$ & $73.5 \%$ & EUR $88.36^{d}$ \\
\hline van Hoek et al. [33] & 75 & Data from van Hoek et al. [72] & Data from van Hoek et al. [72] & $70 \%$ & EUR $86.37^{d}$ \\
\hline van Lier et al. [41] & $60-80$ & Data from van Hoek et al. [72] & Duration of protection of 7.5 years & $75 \%$ & EUR $81.54^{d}$ \\
\hline
\end{tabular}

HZ herpes zoster

a In static models vaccination costs and effects are proportional to vaccination coverage. Hence, the level of coverage has no impact on the incremental cost-effectiveness ratio

bBase-case analysis

Including co-payments

Including administration costs

'This value was assumed for the age group of 60-64 years. The supplemental material of this study also provides values for higher age groups (7-68 \%) but no estimate is given for the age group below 60 years Reimbursement rate was assumed to be $65 \%$ when taking a third-party payer perspective

${ }^{9}$ Costs of an immunisation course comprising two doses

${ }^{\mathrm{h}}$ Including public awareness campaign, patient travel time, time receiving vaccine and costs of treating adverse events 
Table 5 Economic results of the models evaluating routine varicella vaccination (2010 EUR, German price level)

\begin{tabular}{|c|c|c|c|c|c|}
\hline Study & Age at vaccination & $\begin{array}{l}\text { Dose } \\
\text { schedule }\end{array}$ & Comparator & Health care payer perspective & Societal perspective \\
\hline \multirow[t]{3}{*}{ Banz et al. [35] } & 15 months & 1 & No vaccination & BCR 1.75 & BCR 4.12 \\
\hline & $11-12$ years $^{a}$ & 1 & No vaccination & BCR 1.13 & BCR 8.44 \\
\hline & combined & 1 & No vaccination & BCR 1.70 & BCR 4.10 \\
\hline Banz et al. [51] & $1-2$ years & 2 & $\begin{array}{l}\text { 2-dose vaccination } \\
\text { at } 11-15 \text { years }^{\mathrm{a}}\end{array}$ & BCR 0.30; EUR 856/LYG & BCR 1.29 \\
\hline \multirow[t]{2}{*}{ Beutels et al. [37] } & 15 months & 1 & No vaccination & BCR 0.82; EUR 14,700/LYG & BCR 4.60 \\
\hline & 12 years $^{a}$ & 1 & No vaccination & BCR 1.94 & BCR 6.02 \\
\hline \multirow[t]{5}{*}{ Bilcke et al. [30] } & 1 year (95 \% coverage) & 1 & No vaccination & EUR 550-14,140/QALY & NA \\
\hline & $\begin{array}{l}1 \text { year ( } 1 \text { st dose, } 95 \% \text { coverage); } \\
4 \text { years ( } 2 \text { nd dose, } 90 \% \text { coverage) }\end{array}$ & 2 & No vaccination & EUR 5,240-31,942/QALY & NA \\
\hline & $\begin{array}{l}1 \text { year ( } 1 \text { st dose, } 95 \% \text { coverage); } \\
11 \text { years ( } 2 \text { nd dose, } 80 \% \text { coverage) }\end{array}$ & 2 & No vaccination & EUR 5,043-29,775/QALY & NA \\
\hline & $\begin{array}{l}1 \text { year ( } 1 \text { st dose, } 50 \% \text { coverage); } \\
4 \text { years ( } 2 \text { nd dose, } 50 \% \text { coverage) }\end{array}$ & 2 & No vaccination & EUR 3,345-23,240/QALY & NA \\
\hline & $\begin{array}{l}\text { All vaccination options (including and } \\
\text { excluding additional } \mathrm{HZ} \text { vaccination) }\end{array}$ & 2 & No vaccination & $\begin{array}{l}\text { Net QALY loss for many time horizons }{ }^{\mathrm{b}} \text {; } \\
\text { EUR } 36,256-135,961 / \text { LYG }^{\mathrm{b}}\end{array}$ & NA \\
\hline \multirow[t]{2}{*}{ Bonanni et al. [36] } & 12-18 months & 2 & No vaccination & BCR 0.67 & BCR 3.47 \\
\hline & 13 years & 2 & No vaccination & BCR 0.36 & BCR 2.60 \\
\hline \multirow[t]{4}{*}{ Brisson et al. [31] } & 12 months & 1 & No vaccination & BCR 0.61; EUR 38,142/LYG & BCR 5.24 \\
\hline & 12 months & 1 & No vaccination & BCR 0.59; EUR 40,193/LYG ${ }^{c}$ & BCR $5.09^{c}$ \\
\hline & 12 months & 1 & No vaccination & 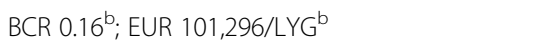 & NA \\
\hline & 12 years $^{\mathrm{a}}$ & 1 & No vaccination & BCR 0.73; EUR 15,863/LYG & BCR 4.44 \\
\hline \multirow[t]{2}{*}{ Brisson et al. [32] } & Infants & 1 & No vaccination & Net QALY loss ${ }^{\mathrm{b}}$ & Net QALY loss ${ }^{\mathrm{b}}$ \\
\hline & 11 years $^{a}$ & 1 & No vaccination & EUR 26,110/QALYb & Cost-saving $^{\mathrm{b}}$ \\
\hline Coudeville et al. [52] & $<6$ years & 1 & No vaccination & Net benefit EUR 326.8 million & NA \\
\hline Coudeville et al. [53] & 12-36 months & 1 & No vaccination & BCR 1.20 at high vaccination coverage & $\begin{array}{l}\text { BCR } 3.50 \text { at high } \\
\text { vaccination coverage }\end{array}$ \\
\hline Coudeville et al. [54] & 12-36 months & 1 & No vaccination & $\begin{array}{l}\text { Cost-saving at high vaccination coverage } \\
\text { (Germany } 51 \% \text {; France } 6.7 \% \text { ); EUR 6.960/LYG } \\
\text { at low vaccination coverage } \\
\text { (France; cost-saving in Germany) }\end{array}$ & $\begin{array}{l}\text { Cost-saving at high } \\
\text { vaccination coverage } \\
\text { (Germany } 61 \% \text {; France } 60 \%)\end{array}$ \\
\hline Dlez Domingo et al. [55] & 15 months & 1 & No vaccination & BCR 0.54 & BCR 1.61 \\
\hline Getsios et al. [56] & 12 months & 1 & No vaccination & EUR 71,722/QALY; EUR 36/varicella case avoided & Cost-saving \\
\hline Ginsberg \& Somekh [57] & 12 months & 1 & No vaccination & BCR 1.63 & BCR 19.33 \\
\hline Hammerschmidt et al. [58] & $\begin{array}{l}11-23 \text { months (including a catch-up } \\
\text { of } 2-17 \text { year olds }{ }^{a} \text { ) }\end{array}$ & $\begin{array}{l}2 \text { ( } 1 \text { dose } \\
\text { for catch-up) }\end{array}$ & $\begin{array}{l}\text { 1-dose vaccination } \\
\text { at } 12-15 \text { years }^{\mathrm{a}}\end{array}$ & BCR 1.08 & BCR 2.56 \\
\hline
\end{tabular}


Table 5 Economic results of the models evaluating routine varicella vaccination (2010 EUR, German price level) (Continued)

\begin{tabular}{|c|c|c|c|c|c|}
\hline Huse et al. [59] & 15 months & 1 & No vaccination & NA & $\begin{array}{l}\text { Cost-saving (net benefit } \\
\text { of EUR } 86.28 \text { per vaccinee) }\end{array}$ \\
\hline Lenne et al. [60] & $1-2$ years & 1 & No vaccination & BCR 0.91; EUR 5,202/LYG & BCR 3.70 \\
\hline Lieu et al. [61] & $<6$ years & 1 & No vaccination & $\begin{array}{l}\text { BCR 0,90; EUR 21,648/LYG; EUR 5.68/varicella } \\
\text { case prevented }\end{array}$ & BCR 5.40 \\
\hline Preblud et al. [62] & 15 months & 1 & No vaccination & BCR 0.30 & $\begin{array}{l}\text { BCR } 6.90 \text { (including home } \\
\text { care costs) }\end{array}$ \\
\hline Scuffham et al. [63] & 15 months & 1 & $\begin{array}{l}\text { No routine vaccination } \\
\text { but low private coverage }\end{array}$ & BCR 0.67 & BCR 2.79 \\
\hline \multirow[t]{2}{*}{ Scuffham et al. [34] } & 12 months & 1 & No vaccination & $\begin{array}{l}\text { EUR 49.11/varicella case avoided; } \\
\text { EUR 16,439/hospitalisation avoided }\end{array}$ & NA \\
\hline & 12 years $^{\mathrm{a}}$ & 1 & No vaccination & $\begin{array}{l}\text { EUR 404.81/varicella case avoided; } \\
\text { EUR 26,791/hospitalisation avoided }\end{array}$ & NA \\
\hline Thiry et al. [64] & 11 years $^{a}$ & 1 & No vaccination & BCR 0.54; EUR 26,988/LYG & BCR 2.17 \\
\hline \multirow[t]{2}{*}{ van Hoek et al. [33] } & 1 year (first dose); 3 years (second dose) & 2 & No vaccination & $\begin{array}{l}41 \% \text { of the simulations below GBP } 20,000 / \mathrm{QALY} \\
\text { (EUR 26,576/QALY) } ; 50 \% \text { of the simulations } \\
\text { below GBP } 30,000 / \mathrm{QALY} \text { (EUR 39,864/QALY) }\end{array}$ & NA \\
\hline & $\begin{array}{l}1 \text { year (first dose); } 3 \text { years (second dose) }+ \\
\mathrm{HZ} \text { vaccination of the elderly }\end{array}$ & 2 & No vaccination & $\begin{array}{l}50 \% \text { of the simulations below GBP } 20,000 / \text { QALY } \\
\text { (EUR 26,576/QALY) } ; 70 \% \text { of the simulations } \\
\text { below GBP 30,000/QALY (EUR 39,864/QALY) }\end{array}$ & NA \\
\hline \multirow[t]{3}{*}{ Zhou et al. [29] } & Children & 1 & No vaccination & BCR 1.00 & BCR 4.37 \\
\hline & Children & 2 & No vaccination & BCR 0.61 & BCR 2.73 \\
\hline & Children & 2 & 1-dose vaccination & BCR 0.13 & BCR 0.56; EUR 95,584/QALY \\
\hline
\end{tabular}

$B C R$ benefit-cost ratio; $L Y G$ life-year gained; $Q A L Y$ quality-adjusted life-year; $N A$ not applicable

${ }^{a}$ With a negative or uncertain history of varicella

Including the impact on $\mathrm{HZ}$

Including the impact of breakthrough varicella 
assuming no exogenous boosting. The authors of this study found that a two-dose vaccination regime would be less cost-effective than a single-dose childhood vaccination strategy. Beyond that, the study by Zhou et al. [29] showed that the choice of the comparator was very influential when evaluating two-dose varicella vaccination. In the study by Bilcke et al. [30] a two-dose vaccination strategy led to more cost-effective results at lower (50 \%) than at higher vaccination coverage (round $90 \%$ ) due to the development of herd protection effects.

The inclusion of the impact on $\mathrm{HZ}$ incidence led to less cost-effective results. Brisson et al. [31] found an ICER of EUR 101,296 per LYG (health care payer perspective) when evaluating one-dose vaccination for toddlers in Canada. Adopting a similar approach for England and Wales, Brisson et al. [32] found a net QALY-loss. In another study from the UK [33], which focused on a 2-dose schedule, $50 \%$ of the simulations exceeded a threshold of GBP 30,000 per QALY (EUR 39,864 per QALY). Bilcke et al. [30] concluded that a childhood vaccination programme is not expected to be cost-effective for several decades when assuming exogenous boosting.

BCRs of vaccinating young adolescents ranged between 0.36 and 1.94 from a health care payer perspective. ICERs were EUR 15.863 per LYG [31], EUR 26,110 per QALY [32] and EUR 26,791 per hospitalisation avoided [34]. Compared to cost-effectiveness estimates of routine childhood varicella vaccination, two studies showed that adolescent vaccination strategies might be less cost-effective than targeting toddlers [35, 36], while other studies found contrary results [31, 32, 34, 37]. The inclusion of indirect costs (societal perspective) improved the BCRs as it was found with the toddler vaccination strategies.

\section{Herpes zoster vaccination}

The results of models assessing the cost-effectiveness of $\mathrm{HZ}$ vaccination are shown in Table 6. One study [38] reported cost-effectiveness results for scenarios most and least in favour of vaccination instead of reporting results of a base-case analysis. Hence, the results of this study comprised a wide range of estimates ranging from EUR 1,200 to 291,240 per QALY (payer perspective). When considering a payer perspective, ICERs among all other studies ranged from EUR 5,412 to 140,125 per QALY. However, the majority of studies reported ICERs from EUR 10,000 to 40,000 per QALY. In terms of costs per HZ case avoided, ICERs varied from EUR 584 to 42,164 in the study from Bilcke et al. [38] and from EUR 817 to 9,433 in other studies when adopting a payer perspective. Few studies also reported ICERs in terms of costs per PHN-case avoided, which ranged from EUR 2,936 to 35,717 (payer perspective).
When taking a societal perspective, one US study [28] reported a wide range of results ranging from costsaving to EUR 250,470 per QALY. Results of other studies varied from EUR 5,628 to 173,224 per QALY.

Several studies, which included waning of vaccineinduced immunity and reported results for different ages at vaccination identified an U-shaped figure of vaccination age-related ICERs: Cost-effectiveness ratios decreased with increasing age at vaccination up to the age of 60 or 70 years and then increased with further increase in age at vaccination. In addition, many studies reported that cost-effectiveness was highly dependent on the assumed duration of vaccine-induced protection and the price of the vaccine. Particularly, the study by Hornberger et al. [28] showed how strongly ICERs can be affected by changes in duration of protection and vaccine cost. Ultsch et al. [39] also found a considerable impact when exploring the combined influence of varying waning immunity rates and durations of stable vaccine efficacy.

The majority of the included studies concluded that $\mathrm{HZ}$ vaccination would represent a cost-effective strategy. However, some studies came to different conclusions, mostly due to the application of different cost-effectiveness thresholds. The authors of a Dutch study [40] concluded that $\mathrm{HZ}$ vaccination might be cost-effective when using a threshold of EUR 50,000 per QALY, but not when decreasing the threshold to EUR 20,000 per QALY. Another study from the Netherlands [41] also reported ICERs above EUR 20,000 per QALY. The authors of this study concluded that $\mathrm{HZ}$ vaccination at the age of 70 years is expected to be marginally cost-effective. Hornberger et al. [28] were very cautious in deriving clear conclusions because of the high uncertainty around the cost-effectiveness results. The same applies to the US study by Rothberg et al. [42]. They found that ICERs often exceeded USD 100,000 (EUR 80,000-90,000) per QALY.

\section{Combined varicella and herpes zoster vaccination strategy}

Two studies [30, 33] modelled the cost-effectiveness of a combined varicella and $\mathrm{HZ}$ vaccination strategy (Table 5). The UK study [33] found that $70 \%$ of the simulations lay below GBP 30,000 (EUR 39,864) per QALY when taking an infinite time horizon. Without the $\mathrm{HZ}$ vaccination component this fraction of simulations decreased to $50 \%$. According to the authors, the combined strategy is likely to be the optimum strategy, but results were highly sensitive with regard to the applied time frame. For example, when adopting time horizons of 30 to 50 years, there was a high probability that the combined strategy would not be cost-effective. The Belgium model adaptation [30], which was based on the previously mentioned UK model, predicted that a combined vaccination strategy would lead to a net QALY loss for many time horizons. 
Table 6 Economic results of the models evaluating HZ vaccination in the elderly (2010 EUR, German price level)

\begin{tabular}{|c|c|c|c|c|}
\hline Study & Age at vaccination (years) & Comparator & Health care payer perspective & Societal perspective \\
\hline \multirow[t]{6}{*}{ Annemans et al. [65] } & $50+$ & No vaccination & $\begin{array}{l}\text { EUR 6,624/QALY; EUR 1,046/HZ case avoided; } \\
\text { EUR 3,495-3,523/PHN case avoided }\end{array}$ & $\begin{array}{l}\text { EUR 6,822/QALY; EUR 1,077/HZ case avoided; } \\
\text { EUR 3,600-3,629/PHN case } \text { avoided }^{\mathrm{a}}\end{array}$ \\
\hline & $60+$ & No vaccination & $\begin{array}{l}\text { EUR 6,809/QALY; EUR 1,310/HZ case avoided; } \\
\text { EUR 3,942-3,969/PHN case avoided }\end{array}$ & $\begin{array}{l}\text { EUR 7,148/QALY; EUR 1,375/HZ case avoided; } \\
\text { EUR 4,039-4,137/PHN case avoided }\end{array}$ \\
\hline & $65+$ & No vaccination & $\begin{array}{l}\text { EUR 7,184/QALY; EUR 1,560/HZ case avoided; } \\
\text { EUR 4,176-4,336/PHN case avoided }\end{array}$ & $\begin{array}{l}\text { EUR 7,577/QALY; EUR 1,645/HZ case avoided; } \\
\text { EUR 4,404-4,574/PHN case } \text { avoided }^{\text {a }}\end{array}$ \\
\hline & $60-64$ & No vaccination & $\begin{array}{l}\text { EUR 5,694/QALY; EUR 817/HZ case avoided; } \\
\text { EUR 2,936-2,969/PHN case avoided }\end{array}$ & $\begin{array}{l}\text { EUR 5,867/QALY; EUR 842/HZ case avoided; } \\
\text { EUR 3,025-3,059/PHN case avoided }\end{array}$ \\
\hline & $65-69$ & No vaccination & $\begin{array}{l}\text { EUR 5,412/QALY; EUR 873/HZ case avoided; } \\
\text { EUR 2,967-2,991/PHN case avoided }\end{array}$ & $\begin{array}{l}\text { EUR 5,628/QALY; EUR 909/HZ case avoided; } \\
\text { EUR 3,087-3,112/PHN case avoided }\end{array}$ \\
\hline & $60-69$ & No vaccination & $\begin{array}{l}\text { EUR 5,553/QALY; EUR 844/HZ case avoided; } \\
\text { EUR 2,951-2,980/PHN case avoided }\end{array}$ & $\begin{array}{l}\text { EUR 5,747/QALY; EUR 874/HZ case avoided; } \\
\text { EUR 3,054-3,085/PHN case avoided }\end{array}$ \\
\hline \multirow[t]{4}{*}{ Bilcke et al. [38] } & 60 & No vaccination & $\begin{array}{l}\text { EUR 1,200-46,968/QALY; } \\
\text { EUR 584-5,148/HZ case avoided }\end{array}$ & NA \\
\hline & 70 & No vaccination & $\begin{array}{l}\text { EUR 2,200-70,496/QALY; } \\
\text { EUR 1,239-8,603/HZ case avoided }\end{array}$ & NA \\
\hline & 80 & No vaccination & $\begin{array}{l}\text { EUR 3,824-126,793/QALY; } \\
\text { EUR 2,867-17,353/HZ case avoided }\end{array}$ & NA \\
\hline & 85 & No vaccination & $\begin{array}{l}\text { EUR 5,272-291,240/QALY; } \\
\text { EUR 4,451-42,164/HZ case avoided }\end{array}$ & NA \\
\hline Bilcke et al. [30] & 50 or 60 & No vaccination & $\begin{array}{l}\text { No results for a sole } \mathrm{HZ} \text { vaccination reported; } \\
\text { see Table } 5 \text { for results on combined varicella } \\
\text { and } \mathrm{HZ} \text { vaccination }\end{array}$ & NA \\
\hline \multirow[t]{2}{*}{ Bresse et al. [66] } & $65+$ & No vaccination & $\begin{array}{l}\text { EUR 11,480/QALY; EUR 2,479/HZ case avoided; } \\
\text { EUR 4,101/PHN case avoided }{ }^{\mathrm{b}}\end{array}$ & NA \\
\hline & $70-79$ & No vaccination & $\begin{array}{l}\text { EUR 8,876/QALY; EUR 2,090/HZ case avoided; } \\
\text { EUR 3,302/PHN case avoided }\end{array}$ & NA \\
\hline \multirow[t]{5}{*}{ Brisson et al. [67] } & 50 & No vaccination & EUR 36,667/QALY & NA \\
\hline & 60 & No vaccination & EUR 26,563/QALY & NA \\
\hline & 65 & No vaccination & EUR 24,002/QALY & NA \\
\hline & 70 & No vaccination & EUR 22,924/QALY & NA \\
\hline & 80 & No vaccination & EUR 33,153/QALY & NA \\
\hline \multirow[t]{4}{*}{ de Boer et al. [40] } & 60 & No vaccination & EUR 40,050/QALY & EUR 33,901/QALY \\
\hline & 65 & No vaccination & EUR 34,440/QALY & EUR 33,511/QALY \\
\hline & 70 & No vaccination & EUR 28,491/QALY & EUR 28,284/QALY \\
\hline & 75 & No vaccination & EUR 28,506/QALY & EUR 28,506/QALY \\
\hline
\end{tabular}


Table 6 Economic results of the models evaluating HZ vaccination in the elderly (2010 EUR, German price level) (Continued)

\begin{tabular}{|c|c|c|c|c|}
\hline Edmunds et al. [68] & 65 & No vaccination & EUR 5,435-100,700/QALYc & NA \\
\hline Hornberger et al. [28] & 69 & No vaccination & NA & From cost-saving up to EUR 250,470/QALYd \\
\hline \multirow[t]{12}{*}{ Moore et al. [69] } & $50+$ & No vaccination & $\begin{array}{l}\text { EUR 17,681/QALY; EUR 1,957/HZ case avoided; } \\
\text { EUR 7,369-7,413/PHN case avoided }\end{array}$ & $\begin{array}{l}\text { EUR 15,520/QALY; EUR 1,710/HZ case avoided; } \\
\text { EUR 6,434-6,472/PHN case avoided }\end{array}$ \\
\hline & $50-54$ & No vaccination & EUR 18,041/QALY & EUR 12,488/QALY \\
\hline & $55-59$ & No vaccination & EUR 16,182/QALY & EUR 12,124/QALY \\
\hline & $60-64$ & No vaccination & EUR 14,931/QALY & EUR 12,866/QALY \\
\hline & $65-69$ & No vaccination & EUR 13,967/QALY & EUR 13,638/QALY \\
\hline & $70-74$ & No vaccination & EUR 17,814/QALY & EUR 17,814/QALY \\
\hline & 75-79 & No vaccination & EUR 20,352/QALY & EUR 20,352/QALY \\
\hline & $80-84$ & No vaccination & EUR 27,176/QALY & EUR 27,176/QALY \\
\hline & $85-89$ & No vaccination & EUR 45,799/QALY & EUR 45,799/QALY \\
\hline & $90-94$ & No vaccination & EUR 67,522/QALY & EUR 67,522/QALY \\
\hline & $95-99$ & No vaccination & EUR 100,562/QALY & EUR 100,562/QALY \\
\hline & $100+$ & No vaccination & EUR 140,125/QALY & EUR 140,125/QALY \\
\hline \multirow[t]{3}{*}{ Najafzadeh et al. [26] } & $60+$ & No vaccination & EUR 28,314/QALY & NA \\
\hline & $60-74$ & No vaccination & EUR 24,002/QALY & NA \\
\hline & $75+$ & No vaccination & EUR 44,123/QALY & NA \\
\hline \multirow[t]{2}{*}{ Pellissier et al. [70] } & $60+$, general population & No vaccination & EUR 16,170/QALY & EUR 14,232/QALY \\
\hline & 60+, immunocompetent only & No vaccination & EUR 24,211/QALY & EUR 22,255/QALY \\
\hline \multirow[t]{6}{*}{ Rothberg et al. [42] } & 60 , male & No vaccination & NA & EUR 130,097/QALY \\
\hline & 60 , female & No vaccination & NA & EUR 81,076/QALY \\
\hline & 70, male & No vaccination & NA & EUR 59,794/QALY \\
\hline & 70, female & No vaccination & NA & EUR 39,512/QALY \\
\hline & 80 , male & No vaccination & NA & EUR 173,224/QALY \\
\hline & 80, female & No vaccination & NA & EUR 111,779/QALY \\
\hline Szucs et al. [71] & $70-79$ & No vaccination & $\begin{array}{l}\text { EUR 13,743/QALY; EUR 3,565/HZ case avoided; } \\
\text { EUR 8,334/PHN case avoided }\end{array}$ & $\begin{array}{l}\text { CHF 15,361/QALY; CHF 3,985/HZ case avoided; } \\
\text { EUR 9,315/PHN case avoided }\end{array}$ \\
\hline
\end{tabular}


Table 6 Economic results of the models evaluating HZ vaccination in the elderly (2010 EUR, German price level) (Continued)

\begin{tabular}{|c|c|c|c|c|}
\hline \multirow[t]{7}{*}{ Ultsch et al. [39] } & 50 & No vaccination & $\begin{array}{l}\text { EUR 37,173/QALY; EUR 1,587/HZ case avoided; } \\
\text { EUR 32,545/PHN case avoided }\end{array}$ & $\begin{array}{l}\text { EUR 30,901/QALY; EUR 1,320/HZ case avoided; } \\
\text { EUR 27,054/PHN case avoided }\end{array}$ \\
\hline & 55 & No vaccination & $\begin{array}{l}\text { EUR 32,480/QALY; EUR 1,518/HZ case avoided; } \\
\text { EUR 26,194/PHN case avoided }\end{array}$ & $\begin{array}{l}\text { EUR 28,244/QALY; EUR 1,320/HZ case avoided; } \\
\text { EUR 22,777/PHN case avoided }\end{array}$ \\
\hline & 60 & No vaccination & $\begin{array}{l}\text { EUR 30,212/QALY; EUR 1,525/HZ case avoided; } \\
\text { EUR 22,337/PHN case avoided }\end{array}$ & $\begin{array}{l}\text { EUR 28,146/QALY; EUR 1,419/HZ case avoided; } \\
\text { EUR 20,809/PHN case avoided }\end{array}$ \\
\hline & 65 & No vaccination & $\begin{array}{l}\text { EUR } 30,807 / \text { QALY; EUR 1,655/HZ case avoided; } \\
\text { EUR 20,951/PHN case avoided }\end{array}$ & $\begin{array}{l}\text { EUR 29,526/QALY; EUR 1,586/HZ case avoided; } \\
\text { EUR 20,079/PHN case avoided }\end{array}$ \\
\hline & 70 & No vaccination & $\begin{array}{l}\text { EUR 42,190/QALY; EUR 2,732/HZ case avoided; } \\
\text { EUR 22,813/PHN case avoided }\end{array}$ & $\begin{array}{l}\text { EUR } 41,942 / \text { QALY; EUR } 2,716 / \mathrm{HZ} \text { case avoided; } \\
\text { EUR 22,679/PHN case avoided }\end{array}$ \\
\hline & 75 & No vaccination & $\begin{array}{l}\text { EUR 55,171/QALY; EUR 3,939/HZ case avoided; } \\
\text { EUR 27,396/PHN case avoided }\end{array}$ & $\begin{array}{l}\text { EUR 54,940/QALY; EUR 3,923/HZ case avoided; } \\
\text { EUR 27,281/PHN case avoided }\end{array}$ \\
\hline & 80 & No vaccination & $\begin{array}{l}\text { EUR 92,734/QALY; EUR 9,433/HZ case avoided; } \\
\text { EUR 35,717/PHN case avoided }\end{array}$ & $\begin{array}{l}\text { EUR 92,541/QALY; EUR 9,414/HZ case avoided; } \\
\text { EUR 35,643/PHN case avoided }\end{array}$ \\
\hline \multirow[t]{4}{*}{ van Hoek et al. [72] } & 60 & No vaccination & EUR 36,302/QALY & NA \\
\hline & 65 & No vaccination & EUR 27,747/QALY & NA \\
\hline & 70 & No vaccination & EUR 20,589/QALY & NA \\
\hline & 75 & No vaccination & EUR 25,211/QALY & NA \\
\hline \multirow[t]{2}{*}{ van Hoek et al. [33] } & 75 & No vaccination & $\begin{array}{l}49 \% \text { of the simulations below GBP 20,000/QALY } \\
\text { (EUR 26,576/QALY); } 96 \% \text { of the simulations below } \\
\text { GBP 30,000/QALY (EUR 39,864/QALY) }\end{array}$ & NA \\
\hline & $\begin{array}{l}75 \text { and } 2 \text {-dose varicella vaccination } \\
\text { of children }\end{array}$ & No vaccination & $\begin{array}{l}50 \% \text { of the simulations below GBP 20,000/QALY } \\
\text { (EUR 26,576/QALY); } 70 \% \text { of the simulations below } \\
\text { GBP 30,000/QALY (EUR 39,864/QALY) }\end{array}$ & NA \\
\hline \multirow[t]{5}{*}{ van Lier et al. [41] } & 60 & No vaccination & EUR 39,577/QALY & EUR 37,638/QALY \\
\hline & 65 & No vaccination & EUR 30,514/QALY & EUR 30,514/QALY \\
\hline & 70 & No vaccination & EUR 21,219/QALY & EUR 21,219/QALY \\
\hline & 75 & No vaccination & EUR 23,779/QALY & EUR 23,779/QALY \\
\hline & 80 & No vaccination & EUR 33,661/QALY & EUR 33,661/QALY \\
\hline
\end{tabular}

HZ herpes zoster; $P H N$ post-herpetic neuralgia; $Q A L Y$ quality-adjusted life-year; $N A$ not applicable

${ }^{\mathrm{a}}$ Depending on the duration of PHN

${ }^{\mathrm{b}}$ All results from the third-party payer perspective

'Depending on the efficacy and the duration of protection

${ }^{\mathrm{d} D e p e n d i n g}$ on vaccination costs 


\section{Discussion}

\section{Key findings}

This systematic review was conducted to summarise the current state of evidence on the cost-effectiveness of varicella and $\mathrm{HZ}$ vaccination in high-income countries. To our knowledge, this is the first systematic review covering cost-effectiveness studies of both varicella and $\mathrm{HZ}$ vaccination. The major findings are outlined below:

\section{Varicella vaccination}

- When ignoring the potential impact on HZ and adopting a health care payer perspective, universal childhood varicella vaccination was usually a costeffective or even cost-saving strategy.

- When switching to a societal perspective, childhood varicella vaccination was found to be a cost-saving intervention.

- Vaccination of adolescents was found to be a costeffective or cost-saving strategy. However, it remains unclear if adolescent vaccination is more or less cost-effective than childhood vaccination due to inconsistent study results.

- Taking the potential impact on HZ into account, it is doubtful that childhood varicella vaccination appears to be cost-effective, at least for several decades.

\section{$H Z$ vaccination}

- In most studies, $\mathrm{HZ}$ vaccination was predicted to be cost-effective or marginally cost-effective.

- When considering both a payer and a societal perspective, the differences in results between the two perspectives decreased with increasing age at vaccination since indirect costs due to sick leave become less relevant in the elderly population.

- When waning of vaccine-induced immunity was modelled, cost-effectiveness of $\mathrm{HZ}$ vaccination was highly dependent on the age at vaccination. ICERs decreased with increasing age at vaccination up to a certain age, followed by a re-increase of the ICERs (U-shape) for older ages. Most results suggest that the optimal age for $\mathrm{HZ}$ vaccination is between 60 and 70 years or around 70 years. Furthermore, costeffectiveness was dependent on the price of the vaccine, the duration of protection and the assumed cost-effectiveness threshold.

\section{Choice of the model}

Model choice can influence the results to a great extent when evaluating the cost-effectiveness of vaccines. In static models such as decision trees and Markov models the force of infection is constant over time because individuals were not allowed to interact with each other. In contrast, dynamic models account for interactions between individuals and therefore the force of infection depends on the number of susceptible, infectious and recovered individuals in the population. This is why dynamic models can include herd protection effects when evaluating the impact of vaccines.

More than half of the studies evaluating varicella vaccination were based on dynamic models and took herd protection effects into account. This is an important requirement to assess the impact of different coverage rates. In several studies the varicella vaccination uptake was found to be one of the most influential parameters. Since the force of infection for $\mathrm{HZ}$ is constant by nature, the models assessing only $\mathrm{HZ}$ vaccination were kept static.

\section{Exogenous boosting}

The results of studies evaluating the cost-effectiveness of varicella vaccination were very sensitive to the structural assumption of allowing for exogenous boosting and its consequences on $\mathrm{HZ}$ incidence. Varicella vaccination was found to be a cost-effective or cost-saving strategy as long as the potential impact on $\mathrm{HZ}$ incidence was ignored. When taking the potential impact on $\mathrm{HZ}$ incidence into account, varicella vaccination was unlikely to be cost-effective. However, the study by van Hoek et al. [33] showed that the negative effect on HZ could, at least partly, be mitigated by the implementation of parallel (temporally limited) $\mathrm{HZ}$ vaccination of the elderly.

\section{Indirect costs}

Many of the studies on varicella vaccination underlined the role of indirect costs for the assessment of costeffectiveness. When adopting a societal perspective, savings were largely due to the inclusion of indirect costs. A previously published review of cost-effectiveness studies on varicella vaccination [43] found that the indirect costs ranged between 42 and $98 \%$ of the total costs. On the contrary, results of models evaluating the costeffectiveness of $\mathrm{HZ}$ vaccination were less sensitive to the cost perspective adopted because in many scenarios the target population belonged to age groups with a low level of labour market participation.

\section{Comparison with previous reviews}

We are aware of four previously published systematic reviews on studies assessing the cost-effectiveness of varicella vaccination [43-46] and one review on studies examining the cost-effectiveness of $\mathrm{HZ}$ vaccination exclusively [47].

Thiry et al. [44] concluded that universal vaccination of healthy children would generate cost savings to society. Although some of the included studies are based on 
dynamic models which could account for herd immunity effects, only one of the (subsequently added) studies covered the potential impact on HZ. The results of this study were only in accordance with the findings of the other studies when ignoring the impact on HZ. Incorporating the impact on $\mathrm{HZ}$ led to highly inefficient results. Rozenbaum et al. [45] found that routine childhood vaccination was a cost-effective or even a costsaving strategy as long as the potential impact on $\mathrm{HZ}$ was not considered in the model analyses. Cost-savings were generally driven by the inclusion of indirect costs in terms of production losses. Rozenbaum et al. [45] attached great importance to point out that the benefits provided by the implementation of early-childhood varicella vaccination might be offset due to an increase in $\mathrm{HZ}$ cases in the elderly population. Therefore, they suggested varicella vaccination of high-risk groups such as susceptible adolescents only, as long as the interactions between varicella and HZ are not clarified. The conclusions of the review by de Soárez et al. [43] were consistent with those of the two previously published reviews $[44,45]$. Interestingly, Unim et al. [46] concluded that the results of the reviewed studies undoubtedly support the introduction of a universal varicella vaccination programme. Although the authors included two studies accounting for the potential impact on HZ [31, 32], the implications of such negative effects of a childhood varicella vaccination programme were not discussed by Unim et al. [46]. Hence, the conclusions of this review are highly disputable. Szucs et al. [47] found that in almost all studies $\mathrm{HZ}$ vaccination was considered as a cost-effective intervention. Amongst others, age at vaccination and vaccination costs had a great impact on the results. This conclusion is in line with findings of our review.

One of the strengths of our systematic review is that it is more comprehensive than previously published review articles. We included economic evaluations of varicella vaccination and $\mathrm{HZ}$ vaccination while other reviews have been focused on one of the two vaccines at a time. A combined assessment of the cost-effectiveness of varicella and HZ vaccination programmes gives consideration to the close relationship of both diseases.

\section{Limitations}

Our review was focused on models evaluating varicella vaccination in children and adolescents as well as $\mathrm{HZ}$ vaccination in the elderly. Studies limited to varicella vaccination of specific target groups such as health care workers or pregnant women were excluded because the current debate is more about whether universal varicella and $\mathrm{HZ}$ vaccination is good value for money; still the consideration of specific risk groups can affect the overall cost-effectiveness. Furthermore, we excluded studies which provided no sufficient description of the used methods (e.g. Hudeckova et al. [48]; Gialoretti et al. [49]); such studies might provide valid results but could not be evaluated. In addition, only one literature database (PubMed) was searched, and our review was restricted to articles written in English or German and to studies reporting results for high-income economies.

It is well known that transferability of results of costeffectiveness analyses across countries is usually affected by a wide range of factors such as variation in disease epidemiology, clinical practice patterns, unit costs, other health care characteristics or methodological decisions [50]. This limitation also holds true for the reviewed studies. For example, there is a large variability in vaccination costs between the included studies. Nevertheless, many of the studies reported similar results and drew consistent conclusions.

\section{Conclusions}

Cost-effectiveness of childhood varicella vaccination rests to a large extent on the interaction between varicella and $\mathrm{HZ}$. When assuming no exogenous boosting of $\mathrm{HZ}$ immunity, varicella vaccination can be considered as a cost-effective or a cost-saving strategy. However, this conclusion needs to be revised when assuming that exogenous boosting exists because the inclusion of the effects of exogenous boosting leads to less favourable results. In this situation, the overall cost-effectiveness seems to become more favourable when routine childhood varicella vaccination is accompanied by the (temporary) implementation of $\mathrm{HZ}$ vaccination in the elderly. As a consequence, clarification on the role of exogenous boosting is crucial for decision-making regarding varicella vaccine introduction. Cost-effectiveness of $\mathrm{HZ}$ vaccination itself is mainly dependent on the chosen age at vaccination, the price of the vaccine and the magnitude of the cost per QALY threshold.

Based on this review we identified several important issues that need to be considered when evaluating the health economic impact of varicella and/or HZ vaccination. Future economic evaluations of varicella vaccination should apply a dynamic modelling approach because only dynamic models can take into account herd protection effects and the potential impact of varicella vaccination on $\mathrm{HZ}$ incidence due to reduced or absent exogenous boosting. In contrast, when the analysis is focused exclusively on $\mathrm{HZ}$ vaccination, a static model seems to be adequate since no change in the force of infection in $\mathrm{HZ}$ will be derived from the introduction of $\mathrm{HZ}$ vaccination. In addition, since fatality rates of varicella and $\mathrm{HZ}$ are rather low, we believe using life years gained (LYG) as an outcome parameter is not sufficient. Thus, we recommend, as done in most existing studies, to consider healthrelated quality of life outcome parameters (e.g. QALYs). 
Moreover, waning of vaccine-induced immunity seems to play an important role for the impact of both vaccines. Hence, we recommend addressing this issue already in the base-case analysis of future evaluations (instead of including waning in sensitivity analysis only). Furthermore, when considering waning of vaccine-induced immunity, the effects of administering a booster vaccine should be captured in sensitivity analysis because a booster shot might have significant impact on the results.

\section{Competing interests}

OD, JH and RTM worked on projects sponsored by Sanofi Pasteur MSD. RTM was a co-applicant for research funding from Sanofi Pasteur MSD. BU was an employee of Sanofi Pasteur MSD (provider of varicella and herpes zoster vaccines) from April 2008 to May 2010. WG and OW declare that they have no competing interests.

\section{Authors' contributions}

$\mathrm{OD}$ and $\mathrm{BU}$ designed the study, conducted the literature search, selected the articles, extracted and interpreted the data, and drafted the manuscript. JH, RTM, WG and OW made significant contributions to the conception of the study, interpretation of the data, and were involved in the critical revision of the manuscript. All authors read and approved the final manuscript.

\section{Acknowledgements}

We acknowledge support of the publication fee by Deutsche

Forschungsgemeinschaft and the Open Access Publication Funds of Bielefeld University.

\section{Author details \\ 'Department of Health Economics and Health Care Management, Bielefeld School of Public Health, Bielefeld University, Bielefeld, Germany. ${ }^{2}$ Immunisation Unit, Robert Koch Institute, Berlin, Germany. ${ }^{3}$ Helmholtz Centre for Infection Research, Brunswick, Germany. ${ }^{4}$ Hannover Medical School, Hannover, Germany.}

Received: 4 February 2014 Accepted: 21 May 2015 Published online: 05 June 2015

\section{References}

1. Miller E, Marshall R, Vurdien J. Epidemiology, outcome and control of varicella-zoster infection. Rev Med Microbiol. 1993;4:222-30.

2. Wutzler P, Färber I, Wagenpfeil S, Bisanz H, Tischer A. Seroprevalence of varicella-zoster virus in the German population. Vaccine. 2002;20:121-4.

3. Schmader K. Herpes zoster and postherpetic neuralgie in older adults. Clin Geriatr Med. 2007;23:615-32.

4. Hope-Simpson RE. Postherpetic neuralgia. J R Coll Gen Pract. 1975;25:571-5.

5. Oster G, Harding G, Dukes E, Edelsberg J, Cleary PD. Pain, medication use, and health-related quality of life in older oersons with postherpetic neuralgia: Results from a population-based survey. J Pain. 2005;6:356-63.

6. Dworkin RH, Portenoy RK. Pain and its persistence in herpes zoster. Pain. 1996;67:241-51.

7. Bonanni P, Breuer J, Gershon A, Gershon M, Hryniewicz W, Papaevangelou V, Rentier B, Rümke H, Sadzot-Delvaux C, Senterre J, Weil-Olivier C, Wutzler P. Varicella vaccination in Europe - taking the practical approach. BMC Med. 2009;7:26.

8. Rentier B, Gershon AA. The Members of the European Working Group on Varicella (EuroVar). Consensus: Varicella vaccination of healthy children. A challenge for Europe. Pediatr Infect Dis J. 2004;23:379-89.

9. Siedler A, Arndt U. Impact of the routine varicella vaccination programme on varicella epidemiology in Germany. Euro Surveill. 2010;15:1-7.

10. Ramet J, Weil-Olivier C, Sedlak W. Is Europe ready to embrace a policy of universal varicella vaccination? Int J Clin Pract. 2005;59:1326-33.

11. Seward JF, Watson BM, Peterson CL, Mascola L, Pelosi JW, Zhang JX, Maupin TJ, Goldman GS, Tabony LJ, Brodovicz KG, Jumaan AO, Wharton M. Varicella disease after introduction of varicella vaccine in the United States, 19952000. JAMA. 2002;287:606-11.
12. Impfplan Österreich 2013. Available at: http://bmg.gv.at/home/Schwerpunkte/ Praevention/Impfen/Oesterreichischer_Impfplan_2013 [accessed 30 September 2013].

13. Advisory Committee on Immunization Practices (ACIP) Recommended Immunization Schedule for Adults Aged 19 Years and Older - United States, 2013. Available at: http://www.cdc.gov/mmwr/preview/mmwrhtml/ su6201a3.htm [accessed 30 September 2013].

14. National Advisory Committee on Immunization. Statement on the recommended use of herpes zoster vaccine. Canada Communicable Disease Report. 2010;36:ACS-1.

15. JCVI short statement on Herpes zoster vaccines. Available at: http://webarchive.nationalarchives.gov.uk/+/www.dh.gov.uk/ab/jcvi/ dh_094744 [accessed 30 September 2013].

16. Hope-Simpson RE. The nature of herpes zoster: A Long-term study and a new hypothesis. Proc R Soc Med. 1965;58:9-20.

17. Bennett GJ, Watson PN. Herpes zoster and postherpetic neuralgia: Past, present and future. Pain Res Manag. 2009;14:275-82.

18. Brisson M, Gay NJ, Edmunds WJ, Andrews NJ. Exposure to varicella boosts immunity to herpes-zoster: implications for mass vaccination against chickenpox. Vaccine. 2002;20:2500-7.

19. van Hoek AJ, Melegaro A, Zagheni E, Edmunds WJ, Gay N. Modelling the impact of a combined varicella and zoster vaccination programme on the epidemiology of varicella zoster virus in England. Vaccine. 2011;29:2411-20.

20. Reynolds MA, Chaves SS, Harpaz R, Lopez AS, Seward JF. The impact of the varicella vaccination program on herpes zoster epidemiology in the United States: A review. J Infect Dis. 2008;197 Suppl 2:224-7.

21. Ogunjimi B, Van Damme P, Beutels P. Herpes zoster risk reduction through exposure to chickenpox patients: A systematic multidisciplinary review. PLos One. 2013;8:e66485.

22. The World Bank: High income: OECD. Available at: http://data.worldbank. org/income-level/OEC [accessed 30 September 2013].

23. Philips Z, Bojke L, Sculpher M, Claxton K, Golder S. Good practice guidelines for decision-analytic modelling in health technology assessment. A review and consolidation of quality assessment. Pharmacoeconomics. 2006;24:355-71.

24. Organisation for Economic Co-operation and Development (OECD): Consumer price indices and purchasing power parities. Available at: http://data.oecd.org/ [accessed 30 September 2013].

25. Moher D, Liberati A, Tetzlaff J, Altman DG, PRISMA Group. Preferred reporting items for systematic reviews and meta-analyses: the PRISMA statement. BMJ. 2009;339:b2535.

26. Najafzadeh M, Marra CA, Galanis E, Patrick DM. Cost effectiveness of herpes zoster vaccine in Canada. Pharmacoeconomics. 2009;27:991-1004.

27. Oxman MN, Levin MJ, Johnson GR, Schmader KE, Straus SE, Gelb LD, Arbeit RD, Simberkoff MS, Gershon AA, Davis LE, Weinberg A, Boardman KD, Williams HM, Hongyuan Zhang J, Peduzzi PN, Beisel CE, Morrison VA, Guatelli JC, Brooks PA, Kauffman CA, Pachucki CT, Neuzil KM, Betts RF, Wright PF, Griffin MR, Brunell P, Soto NE, Marques AR, Keay SK, Goodman RP. A vaccine to prevent herpes zoster and postherpetic neuralgia in older adults. N Engl J Med. 2005;352:2271-84.

28. Hornberger J, Robertus K. Cost-effectiveness of a vaccine to prevent herpes zoster and postherpetic neuralgia in older adults. Ann Intern Med. 2006;145:317-25.

29. Zhou F, Ortega-Sanchez IR, Guris D, Shefer A, Lieu T, Seward JF. An economic analysis of the universal varicella vaccination program in the United States. J Infect Dis. 2008;197 Suppl 2:156-64.

30. Bilcke J, van Hoek AJ, Beutels P. Childhood varicella-zoster virus vaccination in Belgium. Cost-effective only in the long run or without exogenous boosting? Hum Vaccin Immunother. 2013;9:812-22.

31. Brisson M, Edmunds WJ. The cost-effectiveness of varicella vaccination in Canada. Vaccine. 2002;20:1113-25.

32. Brisson M, Edmunds WJ. Varicella vaccination in England and Wales: costutility analysis. Arch Dis Child. 2003;88:862-9.

33. van Hoek AJ, Melegaro A, Gay N, Bilcke J, Edmunds WJ. The costeffectiveness of varicella and combined varicella and herpes zoster vaccination programmes in the United Kingdom. Vaccine. 2012;30:1225-34.

34. Scuffham PA, Lowin AV, Burgess MA. The cost-effectiveness of varicella vaccine programs for Australia. Vaccine. 2000;18:407-145.

35. Banz K, Wagenpfeil S, Neiss A, Goertz A, Staginnus U, Vollmar J, Wutzler P. The cost-effectiveness of routine childhood varicella vaccination in Germany. Vaccine. 2003;21:1256-67. 
36. Bonanni P, Boccalini S, Bechini A, Banz K. Economic evaluation of varicella vaccination in Italian children and adolescents according to different intervention strategies: The burden of uncomplicated hospitalised cases. Vaccine. 2008;26:5619-26.

37. Beutels P, Clara R, Tormans G, van Doorslaer E, van Damme P. Costs and benefits of routine varicella vaccination in German children. J Infect Dis. 1996;174 Suppl 3:335-41.

38. Bilcke J, Marais C, Ogunjimi B, Willem L, Hens N, Beutels P. Costeffectiveness of vaccination against herpes zoster in adults aged over 60 years in Belgium. Vaccine. 2012;30:675-84.

39. Ultsch B, Weidemann F, Reinhold T, Siedler A, Krause G, Wichmann O. Health economic evaluation of vaccination strategies for the prevention of herpes zoster and postherpetic neuralgia in Germany. BMC Health Serv Res. 2013;13:359.

40. de Boer PT, Pouwels KB, Cox JM, Hak E, Wilschut JC, Postma MJ. Costeffectiveness of vaccination of the elderly against herpes zoster in The Netherlands. Vaccine. 2013;31:1276-83.

41. van Lier A, van Hoek AJ, Opstelten W, Boot HJ, de Melker HE. Assessing the potential effects and cost-effectiveness of programmatic herpes zoster vaccination of elderly in the Netherlands. BMC Health Serv Res. 2010;10:237.

42. Rothberg MB, Virapongse A, Smith KJ. Cost-effectiveness of a vaccine to prevent herpes zoster and postherpetic neuralgia in older adults. Clin Infect Dis. 2007:44:1280-8.

43. de Soárez PC, Novaes HMD, Sartori AMC. Impact of methodology on the results of economic evaluations of varicella vaccination programs: is it important for decision-making? Cad Saude Publica. 2009;25 Suppl 3:401-14.

44. Thiry N, Beutels P, van Damme P, van Doorslaer E. Economic evaluations of varicella vaccination programmes. A review of the literature.

Pharmacoeconomics. 2003;21:13-38.

45. Rozenbaum MH, van Hoek AJ, Vegter S, Postma MJ. Cost-effectiveness of varicella vaccination programs: an update of the literature. Expert Rev Vaccines. 2008;7:753-82.

46. Unim B, Saulle R, Boccalini S, Taddei C, Ceccherini V, Boccia A, Bonanni P, La Torre G. Economic evaluation of Varicella vaccination: results of a systematic review. Hum Vaccin Immunother. 2013;9:1932-42.

47. Szucs TD, Pfeil AM. A systematic review of the cost effectiveness of herpes zoster vaccination. Pharmacoeconomics. 2013;31:125-36.

48. Hudečková H, Straka S, Rusňáková S. Epidemiological features and economic evaluation of a potential chickebpox vaccination strategy in Slovac Republic. Cent Eur J Public Health. 2000;8:227-8.

49. Gialoretti LG, Divizia M, Pica F, Volpi A. Analysis of the cost-effectiveness of varicella vaccine programmes based on an observational survey in the latin region of Italy. Herpes. 2005;12:33-7.

50. Goeree R, Burke N, O'Reilly D, Manca A, Blackhouse G, Tarride J-E. Transferability of economic evaluations: approaches and factors to cionsider when using results from one geographic area for another. Curr Med Res Opin. 2007:23:671-82.

51. Banz K, Iseli A, Aebi C, Brunner M, Schmutz AM, Heininger U. Economic evaluation of varicella vaccination in Swiss children and adolescents. Hum Vaccin. 2009;5:847-57.

52. Coudeville L, Paree F, Lebrun T, Sailly J. The value of varicella vaccination in healthy children: cost-benefit analysis of the situation in France. Vaccine. 1999;17:142-51.

53. Coudeville L, Brunot A, Giaquinto C, Lucioni C, Dervaux B. Varicella vaccination in Italy. An economic evaluation of different scenarios. Pharmacoeconomics. 2004;22:839-55.

54. Coudeville L, Brunot A, Szucs TD, Dervaux B. The economic value of childhood varicella vaccination in France and Germany. Value Health. 2005;8:209-22.

55. Díez Domingo J, Ridao M, Latour J, Ballester A, Morant A. A cost benefit analysis of routine varicella vaccination in Spain. Vaccine. 1999;17:1306-11.

56. Getsios D, Caro JJ, Caro G, de Wals P, Law BJ, Robert Y, Lance JR. Instituting a routine varicella vaccination program in Canada: an economic evaluation. Pediatr Infect Dis J. 2002;21:542-7.

57. Ginsberg GM, Somekh E. Cost containment analysis of childhood vaccination against varicella in Israel. J Infect. 2004;48:119-33.

58. Hammerschmidt T, Bisanz H, Wutzler P. Universal mass vaccination against varicella in Germany using an MMRV combination vaccine with a two-dose schedule: An economic analysis. Vaccine. 2007;25:7307-12.

59. Huse DM, Meissner HC, Lacey MJ, Oster G. Childhood vaccination against chickenpox: An analysis of benefits costs. J Pediatr. 1994;124:869-74.

60. Lenne X, Diez Domingo J, Gil A, Ridao M, Lluch JA, Dervaux B. Economic evaluation of varicella vaccination in Spain - Results from a dynamic model. Vaccine. 2006;24:6980-9.
61. Lieu TA, Cochi SL, Black SB, Halloran E, Shinefield HR, Holmes SJ, Wharton $M$, Washington E. Cost-effectiveness of a routine varicella vaccination program for US children. JAMA. 1994;271:375-81.

62. Preblud SR, Orenstein WA, Koplan JP, Bart KJ, Hinman AR. A benefit-cost analysis of a childhood varicella vaccination programme. Postgrad Med J. 1985;61 Suppl 4:17-22

63. Scuffham P, Devlin N, Eberhardt-Phillips J, Wilson-Salt R. The costeffectiveness of introducing a varicella vaccine to the New Zealand immunisation schedule. Soc Sci Med. 1999;49:763-79.

64. Thiry N, Beutels P, Tancredi F, Romanò L, Zanetti A, Bonanni P, Gabutti G, van Damme $P$. An economic evaluation of varicella vaccination in Italian adolescents. Vaccine. 2004;22:3546-62.

65. Annemans L, Bresse X, Gobbo C, Papageorgiou M. Health economic evaluation of a vaccine for the prevention of herpes zoster (shingles) and postherpetic neuralgia in adults in Belgium. J Med Econ. 2010;13:537-51.

66. Bresse X, Annemans L, Préaud E, Bloch K, Duru G, Gauthier A. Vaccination against herpes zoster and postherpetic neuralgia in France: a costeffectiveness analysis. Expert Rev Pharmacoecon Outcomes Res. 2013;13:393-406.

67. Brisson M, Pellissier JM, Camden S, Quach C, de Wals P. The potential costeffectiveness of vaccination against herpes zoster and post-herpetic neuralgia. Hum Vaccin. 2008;4:238-45.

68. Edmunds WJ, Brisson M, Rose JD. The epidemiology of herpes zoster and potential cost-effectiveness of vaccination in England and Wales. Vaccine. 2001;19:3076-90.

69. Moore L, Remy V, Martin M, Beillat M, McGuire A. A health economic model for evaluating a vaccine for the prevention of herpes zoster and postherpetic neuralgia in the UK. Cost Eff Resour Alloc. 2010;8:7.

70. Pellissier JM, Brisson M, Levin MJ. Evaluation of the cost-effectiveness in the United States of a vaccine to prevent herpes zoster and postherpetic neuralgia in older adults. Vaccine. 2007;25:8326-37.

71. Szucs TD, Kressig RW, Papageorgiou M, Kempf W, Michel J, Fendl A, Bresse $X$. Economic evaluation of a vaccine for the prevention of herpes zoster and post-herpetic neuralgia in older adults in Switzerland. Hum Vaccin. 2011;7:749-56

72. van Hoek AJ, Gay N, Melegaro A, Opstelten W, Edmunds WJ. Estimating the cost-effectiveness of vaccination against herpes zoster in England and Wales. Vaccine. 2009;27:1454-67.

\section{Submit your next manuscript to BioMed Central and take full advantage of:}

- Convenient online submission

- Thorough peer review

- No space constraints or color figure charges

- Immediate publication on acceptance

- Inclusion in PubMed, CAS, Scopus and Google Scholar

- Research which is freely available for redistribution

Submit your manuscript at www.biomedcentral.com/submit

C Biomed Central 